\title{
Antioxidant and Antibacterial Profiling of Pomegranate-pericarp Extract Functionalized-zinc Oxide Nanocomposite
}

\author{
Mahendra Singh, Kyung Eun Lee, Ramachandran Vinayagam, and Sang Gu Kang
}

Received: 29 July 2021 / Revised: 29 August 2021 / Accepted: 2 September 2021

(C) The Korean Society for Biotechnology and Bioengineering and Springer 2021

\begin{abstract}
With the advancement in green nanotechnology, considerable attention is being given to the synthesis of different kinds of nanomaterials for biological applications. In this study, zinc oxide nanocomposites (ZnO NPs) were synthesized using Punica granatum L. (Pomegranate) pericarp ethanolic extract (PE) by the chemical precipitation method. The prepared $\mathrm{ZnO}$ NPs showed a characteristic peak at 270 $\mathrm{nm}$ in the UV-Vis spectrophotometer and chemical bond stretching in the Fourier transforms infrared spectroscopy (FT-IR) spectra, indicated the formation of PE-functionalized zinc oxide nanocomposite (PE-ZnO NPs). The SEM results showed agglomerated PE-ZnO NPs of a spherical shape with an average size of $80-100 \mathrm{~nm}$. Moreover, biological assessment of the PE-ZnO NPs revealed significant scavenging activity in DPPH (116.5\%) and $\mathrm{ABTS}^{+}(95.2 \%)$ radical assay methods, and substantial antibacterial activity against Bacillus cereus, Bacillus licheniformis, and Escherichia coli. Furthermore, PE-ZnO NPs showed about $96.3 \%$ of cell viability for human $\mathrm{HaCaT}$ cells at the maximum concentration $(100 \mu \mathrm{g} / \mathrm{mL})$, marked as a reliable bioactive agent. Therefore, the developed PE-ZnO NPs were elucidated with substantial ROS scavenger and non-antibiotic antibacterial agent and hence, can be applied in respective biological applications.
\end{abstract}

Mahendra Singh, Kyung Eun Lee, Ramachandran Vinayagam*, Sang Gu Kang $^{*}$

Department of Biotechnology, College of Life and Applied Sciences, Yeungnam University, Gyeongsan 38541, Korea

E-mail: rambio85@gmail.com

Tel: +82-53-810-3025

E-mail: kangsg@ynu.ac.kr

Kyung Eun Lee

Stemforce, 313 Institute of Industrial Technology, Yeungnam University, Gyeongsan 38541, Korea
Keywords: Pomegranate, antioxidant, antibacterial activities, zinc oxide nanocomposite, cytotoxicity

\section{Introduction}

Nanotechnology is an emergent study area of advanced science and technology because of its several multifunctional applications. Nanoparticles (NPs) refer to particles with about $100 \mathrm{~nm}$ and less than one-tenth of a million meters, multiple morphologies, and other physicochemical properties [1]. The NPs are used in various applications in many fields; for example, medicine, environment, agriculture, cosmetics, and diagnostic fields [2]. NPs are classified into four types: polymer, carbon, metal and metal-oxide, and other inorganic substances. Amongst the multiple forms of NPs, metal-oxide NPs have been used more frequently on humans and biological applications because of less toxicity [3].

Among the various metal oxides: zinc-oxide nanoparticles ( $\mathrm{ZnO}$ NPs) is widely used in many application, including environmental remediation, piezoelectric, electronics, sensor, solar energy conversion, drug delivery, light-emitting diode, cosmetics, and antimicrobial products [4]. Moreover, the $\mathrm{ZnO}$ NPs have many advantages as a modern drug delivery system because of easy fabrication, low production cost, tunable structure, high drug-loading capability, biocompatibility, controlled drug-release ability, and target delivery $[5,6]$. The $\mathrm{ZnO}$ NPs are also being used to produce a white appearance and UV-blocking material in textiles [7]. Besides, the ZnO NPs can easily engage on the bacterial cell wall and lead to membrane damage [8]. In recent years, $\mathrm{ZnO}$ NPs are also applied as external antibacterial agents in food packaging, preparation of ointments, cloth fabrics, lotion, and mouthwash to avert microbial load [9]. 
Nano cosmetics are cosmetics in a formulation in which bio nanoparticles or structures such as zinc oxide nanoparticles of about $100 \mathrm{~nm}$ can be used as raw materials [10]. In addition, nanomaterials are used to develop high-valueadded cosmetics that increase skin health promotion effects by blocking fine dust, ultraviolet rays, infrared rays, and harmful rays or chemicals from outside [11]. In this context, the $\mathrm{ZnO}$ NPs have been used in cosmetics, such as sunscreens, to protect the skin from harmful radiations [12]. Moreover, the food drug administration (FDA, USA) accepts $\mathrm{ZnO}$ as a GRAS (Generally Recognized As Safe) material compared to another metal nanomaterial [13].

The sunscreen comes in a range of colors, including colorless and powder. Materials such as aminobenzoic acid, acid salicylates, cinnamates, and benzoic acid are used in the structure of the sunscreens. Recent trends in cosmetics ingredients use plant-derived natural compounds. For these natural-oriented trends in cosmetics, natural preservatives mainly used plant extracts that have antioxidant, antibacterial, and photoprotective effects [14]. Recently, green synthesis of $\mathrm{ZnO}$ NPs has been reported using orange fruit and peel extracts for antibacterial activities [15-17]. Since fruits have abundant beneficial phytochemicals, including alkaloids, flavonoids, terpenoids, carotenoids, and tannins, plant fruits can be used as nanocomposite agents to synthesize NPs materials [18].

Punica granatum L. (Pomegranate) is an edible fruit that belongs to the family Punicaceae (now in the Lythraceae family) [19]. Pomegranate fruit is rich in natural polyphenols, flavonoids, and proanthocyanidins, and commonly consumed as nutritional food, for therapeutic activities, promoting health, and high anti-oxidant function [20]. The pomegranate seeds have been recognized to scavenge the reactive oxygen species (ROS), as it contains vitamin C, vitamin K, folic acid, and polyphenol-rich bioactive compounds. Furthermore, pomegranate fruit pericarp mainly contains potent bioactive compounds, such as gallagic, ellagic acid, ellagitannins, punicalagin, anthocyanins delphinidin, pelargonidin, and luteolin [21,22], while pomegranate fruit peel was estimated to mainly contain $25-28 \%$ of the polyphenols with gallic acid and tannins for scavenging the ROS (reactive oxygen species) [23]. Moreover, many studies reported that pomegranate seed juices and peel extracts are potentially beneficial for treating diabetes [24], obesity [25], cardiovascular disease [26,27], and inflammation [28,29]. In addition, pomegranate fruit extract has anti-cancer properties and alternative therapy for prostate cancer [30,31]. In particular, the primary substance of the polyphenol of pomegranate fruit has antimicrobial action against Klebsiella pneumonia, Escherichia coli, and Pseudomonas aeruginosa [32]. Hence, we synthesized novel PE-ZnO NPs with pomegranate ethanol extract (PE) for antioxidant and antibacterial activities as well as studied for the cytotoxicity in vitro using human keratinocyte cell lines.

\section{Material and Methods}

\subsection{Chemicals}

Folin-Ciocalteu, 1,1-diphenyl-2-picryl hydrazyl (DPPH), 2, 2'-azinobis(3-ethylbenzothiazoline-6-sulfonic acid) diammonium salt (ABTS), and Methyl thiazolyl diphenyl-tetrazolium bromide (MTT) were obtained from Sigma Aldrich CO. (St. Louis, USA) and Duksan (Ansan, Korea). The fetal bovine serum (FBS), Dulbecco's modified Eagle's medium (DMEM), and trypsin-disodium ethylenediamine tetra-acetic acid (EDTA) were procured from Welgene (Gyeongsan, Korea). Phosphate buffered saline (PBS) and penicillin $(100$ units $/ \mathrm{mL}) /$ streptomycin $(100 \mu \mathrm{g} / \mathrm{mL})$ were obtained from HyClone (Logan, USA). All other chemicals and reagents of analytical grade were used in this present study.

\subsection{Ethanol extract of pomegranate pericarp}

The healthy pomegranate fruits were harvested at the Yeungnam University farm in Gyeongsan, Korea and washed with tap water. Next, the pomegranate fruits were cut manually, and pericarps were dried at room temperature; then, grounded using an electronic grinder to a coarse powder and stored in an incubator at $4^{\circ} \mathrm{C}$. To prepare a sample, $50 \mathrm{~g}$ of grounded pericarp was soaked in $500 \mathrm{~mL}$ of $70 \%$ ethanol and stored at room temperature for $48 \mathrm{~h}$. After this, the samples were centrifuged at $8000 \times g$ for $10 \mathrm{~min}$, the collected supernatant was filtered with $0.4 \mu \mathrm{m}$ of filter paper (Whatman No.1) and dried by employing a rotary evaporator at $60^{\circ} \mathrm{C}(\mathrm{N}-1000$, Eyela, Tokyo, Japan). At the end of the extraction, the collected dried extract was stored in sterile bottles in the incubator at $4^{\circ} \mathrm{C}$. The pooled pomegranate extract (PE) was then used for the analysis of phenolics, antioxidant, and antibacterial activities.

\subsection{Determination of total polyphenolic and flavonoid content}

Initially, the collected PE was assessed for the total phenolic content using the Folin-Ciocalteu reagents [33]. Briefly, $1 \mathrm{~g}$ test sample (PE) was mixed with $10 \mathrm{~mL}$ of distilled water and centrifugation at $240 \times g$ for $10 \mathrm{~min}$. The collected supernatant was filtered with Whatman No.1, and the filtrate was further centrifuged at $8000 \times g$ for 10 min. Next, $200 \mu \mathrm{L}$ PE was mixed with $670 \mu \mathrm{L}$ distilled water followed by the addition of $600 \mu \mathrm{L}$ sodium carbonate (2\%) and $30 \mu \mathrm{L}$ of Folin-Ciocalteu (50\%) reagent. The complete reaction mixture was incubated at room temperature for $30 \mathrm{~min}$ and measured for absorbance at $765 \mathrm{~nm}$ using a UV-Vis spectrophotometer (Optizen 
2120UV, Mecasys, Daejeon, Korea). Besides, gallic acid (Sigma Aldrich Co., St. Louis, USA) was used as standard phenol to plot a calibration curve $(\mathrm{y}=0.0003 \mathrm{x}+0.1094$, $\left.\mathrm{R}^{2}=0.9989\right)$ for the calculation of the total phenolic content in PE and expressed by gallic acid equivalents ( $\mathrm{mg}$ GAE/g extract).

Moreover, the total flavonoid content was also measured using the previously reported method [33]. Briefly, $200 \mu \mathrm{L}$ of the collected PE was mixed with a solution mixture of $790 \mu \mathrm{L}$ distilled water, $10 \%$ aluminum chloride hexahydrate (AlCl3), $30 \mu \mathrm{L} 1 \mathrm{M}$ potassium acetate $\left(\mathrm{CH}_{3} \mathrm{COOK}\right)$, and $450 \mu \mathrm{L} \mathrm{95 \%} \mathrm{ethanol} \mathrm{and} \mathrm{mixed} \mathrm{well.} \mathrm{The} \mathrm{reaction} \mathrm{mixture}$ was incubated at room temperature for $30 \mathrm{~min}$ and measured for absorbance at $510 \mathrm{~nm}$ using a UV/Vis spectrophotometer (Optizen 2120UV, Mecasys, Daejeon, Korea). Also, quercetin was employed as a standard flavonoid to plot a standard curve $(y=0.0024 x+0.0055, R 2=0.9976)$ for the calculation of the total flavonoid concentration in PE and expressed as quercetin equivalents (mg QE/g extract).

\subsection{Synthesis and characterization of PE-ZnO NPs}

The synthesis of pomegranate-pericarp extract functionalizedzinc oxide nanocomposite (PE-ZnO NPs) was carried out using the chemical precipitation method. Briefly, $0.2 \mathrm{~g}$ of PE was dissolved in $200 \mathrm{~mL}$ of deionized water and amended with $1 \mathrm{~mL}$ of $1 \mathrm{M}$ zinc nitrate (Sigma Aldrich), incubated under shaking conditions at room temperature. Next, $500 \mathrm{~mL}$ of $2 \mathrm{M}$ of sodium borohydride was added dropwise to the above solution under vigorous shaking conditions at room temperature for $4 \mathrm{~h}$. Finally, synthesized PE-ZnO NPs were harvested from the solution using centrifugation at 2,500 $\times g$ under room temperature and washed thrice with deionized water by centrifugation. The synthesized NPs were then dried in the incubator at $40^{\circ} \mathrm{C}$ and used in further experimental analysis. Under similar conditions, only $\mathrm{ZnO}$ NPs were also prepared and used as a reference control for the PE-ZnO NPs.

The synthesized PE-ZnO NPs were examined for the nanocomposite formation by monitoring the metallic plasmonic peaks in the UV-Vis spectra calculated using a UV/Vis spectrophotometer (Optizen 2120UV, Mecasys, Daejeon, Korea). Moreover, the functionalization of $\mathrm{PE}$ on the $\mathrm{ZnO}$ NPs was also evaluated in terms of transmittance (400 to $4,000 \mathrm{~cm}^{-1}$ range) using the Fourier TransformInfrared spectrometer (FT-IR, PerkinElmer, Inc., Waltham, USA). Next, the morphology of the synthesized PE-ZnO NPs was also visualized using Scanning Electron Microscope (SEM), where $10 \mu \mathrm{L}$ of $0.2 \%$ (w/v) of PE-ZnO NPs solution was dropped on the carbon tape and air-dried. Finally, the samples were coated with platinum $(\mathrm{Pt})$ and visualized under SEM S4800, Hitachi, Ltd.
2.5. $\mathrm{ABTS}^{++}$and DPPH scavenging activity measurement The antioxidant activity of the synthesized PE-ZnO NPs was monitored by $\mathrm{ABTS}^{+}$free radical scavenging method as reported earlier [34]. Herein, $7 \mathrm{mM}$ ABTS and $2.5 \mathrm{mM}$ potassium persulfate were mixed in equal volume and stored in dark under ambient temperature for $24 \mathrm{~h}$ to form $\mathrm{ABTS}^{+}$radicals. Next, this solution was diluted 2 times with ethanol and measured for absorbance at $734 \mathrm{~nm}$ using an ELISA reader (InfiniteTM F200, Männedorf, Switzerland). To monitor the scavenging activity of PE-ZnO NPs, equal volume $(150 \mu \mathrm{L})$ of ABTS solution and the $0.2 \%$ test sample was mixed well, allowed to react for $6 \mathrm{~min}$ at room temperature, and measured for the absorbance at $734 \mathrm{~nm}$ using an ELISA reader (InfiniteTM F200, Männedorf, Switzerland).

The DPPH free radical scavenging activity was also assessed for the PE-ZnO NPs as previously reported [35]. Briefly, $200 \mu \mathrm{L}$ of the $0.2 \%$ PE-ZnO NPs was mixed with $100 \mu \mathrm{L}$ of $0.2 \mathrm{mM} \mathrm{DPPH}$ solution in ethanol, allowed to react for $30 \mathrm{~min}$ at room temperature, and measured for absorbance at $517 \mathrm{~nm}$ using ELISA reader (Infinite $^{\mathrm{TM}}$ F200, Männedorf, Switzerland). Besides, ethanol was treated instead of DPPH or $\mathrm{ABTS}^{+}$as negative control and ascorbic acid was employed as a positive control. The scavenging activity of the test sample was calculated using Eq. 1.

Scavenging rate $(\%)=\frac{\mathrm{OD}(\text { control })-\mathrm{OD}(\text { sample })}{\mathrm{OD}(\text { control })} \times 100 \%$

Further, the PE and $\mathrm{ZnO}$ NPs were also considered under similar experimental conditions for the $\mathrm{ABTS}^{+}$and DPPH scavenging activity as reference controls against the PE-ZnO NPs.

\subsection{Cell culture}

Human keratinocytes ( $\mathrm{HaCaT}$ ) cell line was obtained from American type culture collection (ATCC) Manassas, VA, USA. The HaCaT cells were grown and maintained in Dulbecco's Modified Eagle Medium (DMEM) amended with $10 \%$ FBS, penicillin (100 units/mL)/streptomycin 100 $\mu \mathrm{g} / \mathrm{mL}$ ) under $5 \% \quad \mathrm{CO}_{2}$ atmosphere at $37^{\circ} \mathrm{C}$ in a $\mathrm{CO}_{2}$ incubator. The cell lines were cultured with undersupply and subculture was performed at 2-3 days intervals.

\subsection{Cytotoxicity and proliferation test}

The cytotoxicity and proliferation inhibition induced by the synthesized PE-ZnO NPs were also assessed by MTT assay. Briefly, the HaCaT cells were harvested $\left(1 \times 10^{4}\right.$ cells/well) and seeded in 96 well cell culture plates and incubated with growth for $24 \mathrm{~h}$. Following, old medium was replaced with a new growth medium amended with 
various concentrations $(25,50$, and $100 \mathrm{mg} / \mathrm{mL})$ of $\mathrm{PE}$, $\mathrm{ZnO} \mathrm{NPs}$, and PE-ZnO NPs and further incubated under $5 \% \mathrm{CO}_{2}$ atmosphere at $37^{\circ} \mathrm{C}$ for $48 \mathrm{~h}$ in a $\mathrm{CO}_{2}$ incubator. Following, the cell Titer $96^{\circledR}$ AQueous One Solution Cell Proliferation Assay Kit (Promega, USA) was employed to monitor the effect on sample treatment on cell proliferation. Herein, all the medium from the treated cells was replaced with fresh $100 \mu \mathrm{L}$ of DMEM followed by the addition of $20 \mu \mathrm{L}$ of MTT solution and gently mixed. This reaction mixture was then incubated under a $5 \% \mathrm{CO}_{2}$ atmosphere at $37^{\circ} \mathrm{C}$ in a $\mathrm{CO}_{2}$ incubator and measured the absorbance at $490 \mathrm{~nm}$ by employing an ELISA reader (InfiniteTM F200, Männedorf, Switzerland). An untreated cell culture set was employed as a control.

\subsection{Antimicrobial activity}

Bacterial strains, i.e., Bacillus licheniformis (ATCC 1458), Bacillus cereus (ATCC 14579), and E. coli (ATCC 15597), were purchased from the ATCC (American Type Culture Collection, Manassas, Virginia, USA) and used for the antibacterial activity assay with experimental samples, i.e., synthesized PE-ZnO NPs, ZnO NPs, and PE (0.1 and 1.0 $\mathrm{mg} / \mathrm{mL}$ ), against Tetracycline as a positive control. Herein, $10 \mu \mathrm{L}$ of each bacterial culture was inoculated from broth culture in $10 \mathrm{~mL} \mathrm{LB}$ (Luria-Bertani) media and incubated for $12 \mathrm{~h}$ under shaking condition $(120 \mathrm{rpm})$ at $37^{\circ} \mathrm{C}$. Following bacterial cells were harvested and washed with PBS (pH 6.8) to obtain $10^{4}$ cells $/ \mathrm{mL}$. Next, these cells were inoculated in fresh LB media amended with various concentrations of sample and further incubated under shaking conditions at $37^{\circ} \mathrm{C}$ for $12 \mathrm{~h}$. After that, treated bacterial cultures were serially diluted $\left(10^{-5}-10^{-7}\right)$ and gently speeded on the LB agar media plates, incubated at $32^{\circ} \mathrm{C}$ for $12 \mathrm{~h}$. Finally, colonies appeared on the agar plates were counted to calculate the antibacterial activity induced by experimental samples.

\subsection{Statistical analysis}

All data was calculated as Mean \pm S.D values using SPSS package (SPSS, USA). Statistical analysis of the experimental data made the significance of the experimental group relative to the control group as student's t-test, and when the value of $p<0.05$.

\section{Results and Discussion}

In this study, pomegranate pericarp extracts functionalized ZnO NPs (PE-ZnO NPs) were synthesized, characterized, and examined for biological properties. It was hypothesized that bioactive ingredients in PE, i.e., polyphenols and flavonoids, can be decorated on the growing clusters of $\mathrm{Zn}^{+2}$ ions in presence of a reducing agents to form PE- $\mathrm{ZnO}$ NPs. The bioactive compounds profiling revealed considerable content of polyphenols and flavonoids in PE which can be used in the synthesis of PE-ZnO NPs.

\subsection{Total polyphenolic content assay of PE extracts}

The PE fruit is enriched with bioactive polyphenols and is commonly consumed in large quantities around the world as health functional foods [36]. However, pomegranate pericarp is usually discarded as fruit waste. Therefore, in this study, pomegranate pericarp was evaluated for the total bioactive content and used in the synthesis of PE- ZnO NPs. Initially, the bioactive compounds in the PE were extracted using 70\% ethanol and analyzed for the total phenolic and flavonoid contents. Herein, PE demonstrated significant quantities of polyphenol $(363.04 \pm 0.10 \mathrm{mg}$ $\mathrm{GAE} / \mathrm{g})$ and flavonoid $(20.17 \pm 0.01 \mathrm{mg} \mathrm{QE} / \mathrm{g})$ (Table 1). Therefore, the PE sample may be considered for biological functions by comparison to quercetin, which is a physiologically active substance [18]. Moreover, recent studies have also reported high content of polyphenolic compounds, including punicalagin isomer, anthocyanins (cyanidin, delphinidin, and pelargonidin 3-glucoside and 3,5-diglucoside), ellagic acid, flavonoids, phenolic acids, and other polyphenolic substances in ethanol extract of PE [37]. Due to excellent polarity, ethanol fraction can elute a higher amount of phenolic and flavonoid contents from the extract, and these results were also supported by our finding as reported previously $[38,39]$.

\subsection{Physical properties of PE-ZnO NPs}

The synthesis of PE-ZnO NPs was performed from PE and zinc nitrate as an ingredient by chemical precipitation method and the formation of nanocomposite material was examined using UV-Vis spectra (Fig. 1A). The PE shows maximum absorbance at 310 and $380 \mathrm{~nm}$, respectively while PE-ZnO NPs showed at broad peaks in the region of

Table 1. Total polyphenolic and flavonoids contents of Punica granatum extraction

\begin{tabular}{ccc}
\hline Extracts & $\begin{array}{c}\text { Total Polyphenol } \\
(\mathrm{mg} \mathrm{GAE} / \text { extract g) }\end{array}$ & $\begin{array}{c}\text { Total flavonoid } \\
\left(\mathrm{mg} \mathrm{QE}^{\#} / \mathrm{extract} \mathrm{g}\right)\end{array}$ \\
\hline Punica granatum & $363.04 \pm 0.10^{\mathrm{a}}$ & $20.17 \pm 0.01^{\mathrm{a}}$ \\
\hline
\end{tabular}

${ }^{\#}$ GAE: gallic acid equivalents; ${ }^{\#} \mathrm{QE}$ : quercetin equivalents. ${ }^{\mathrm{a}} \mathrm{Mean} \pm \mathrm{SD}(\mathrm{n}=3)$. 


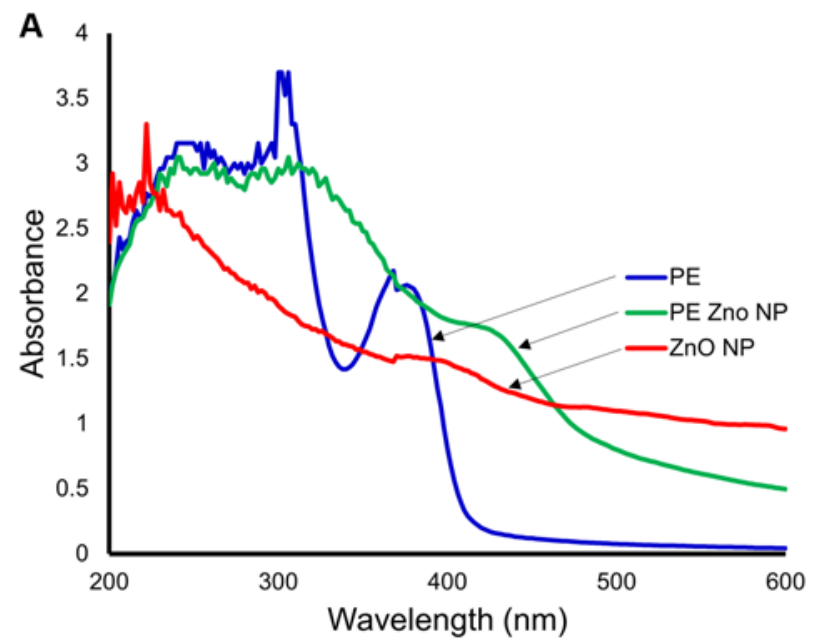

Fig. 1. (A) UV absorption spectrum of PE, ZnO NPs, and PE-ZnO NPs.

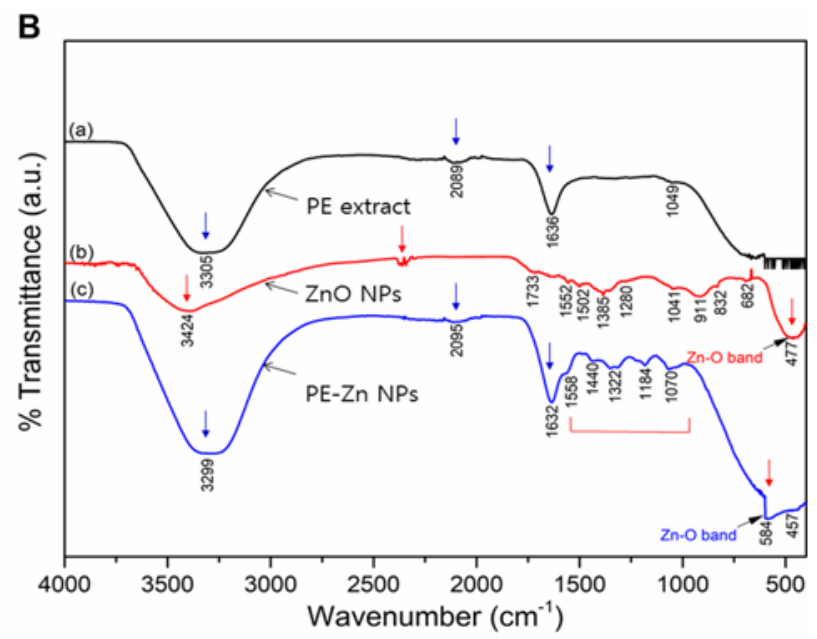

(B) The FT-IR spectra analysis of PE, ZnO NPs, and PE-ZnNP.
250-480 nm which overlaps with the UV-Vis spectra of $\mathrm{ZnO}$ NPs as reported previously [40].

Furthermore, FT-IR spectroscopy was performed to identify the functional groups related to $\mathrm{PE}, \mathrm{ZnO} \mathrm{NPs}$, and PE-ZnO NPs (Fig. 1B). The PE shows a characteristic transmission infrared wavelength band of $3,305 \mathrm{~cm}^{-1}$ for carboxylic acid $\mathrm{O}-\mathrm{H}$ strain, 2,089 $\mathrm{cm}^{-1}$ to aromatic $\mathrm{C}-\mathrm{H}$ folded, 1,636 $\mathrm{cm}^{-1}$ for aromatic ring vibration, and 1,049 $\mathrm{cm}^{-1}$ for primary alcohol C-O stretching of the functional molecules. Likewise, transmission spectrum peaks at
$3,424,1,733,1,552,1,502,911$, and $1,280 \mathrm{~cm}^{-1}$ indicated the formation of ZnO NPs. Analysis of the PE-ZnO NPs showed peaks at 3,299 and $2,095 \mathrm{~cm}^{-1}$ for O-H stretch (phenols) and $\mathrm{N}=\mathrm{C}=\mathrm{S}$ stretch (isothiocyanate) regions, respectively. Also, the bands at 1,632 and $1,558 \mathrm{~cm}^{-1}$ correspond to amine I \& II regions, $1,440 \mathrm{~cm}^{-1}$ for C-C stretch (aromatics), 1,322 $\mathrm{cm}^{-1} \mathrm{~N}-\mathrm{O}$ symmetric stretch (nitro compounds), $1,184 \mathrm{~cm}^{-1}$ for C-N stretch (aliphatic amines), $1,070 \mathrm{~cm}^{-1}$ for $=\mathrm{C}-\mathrm{H}$ bond (alkenes), indicated the functionalization of ZnO NPs (strong bands at $584-457 \mathrm{~cm}^{-1}$ )
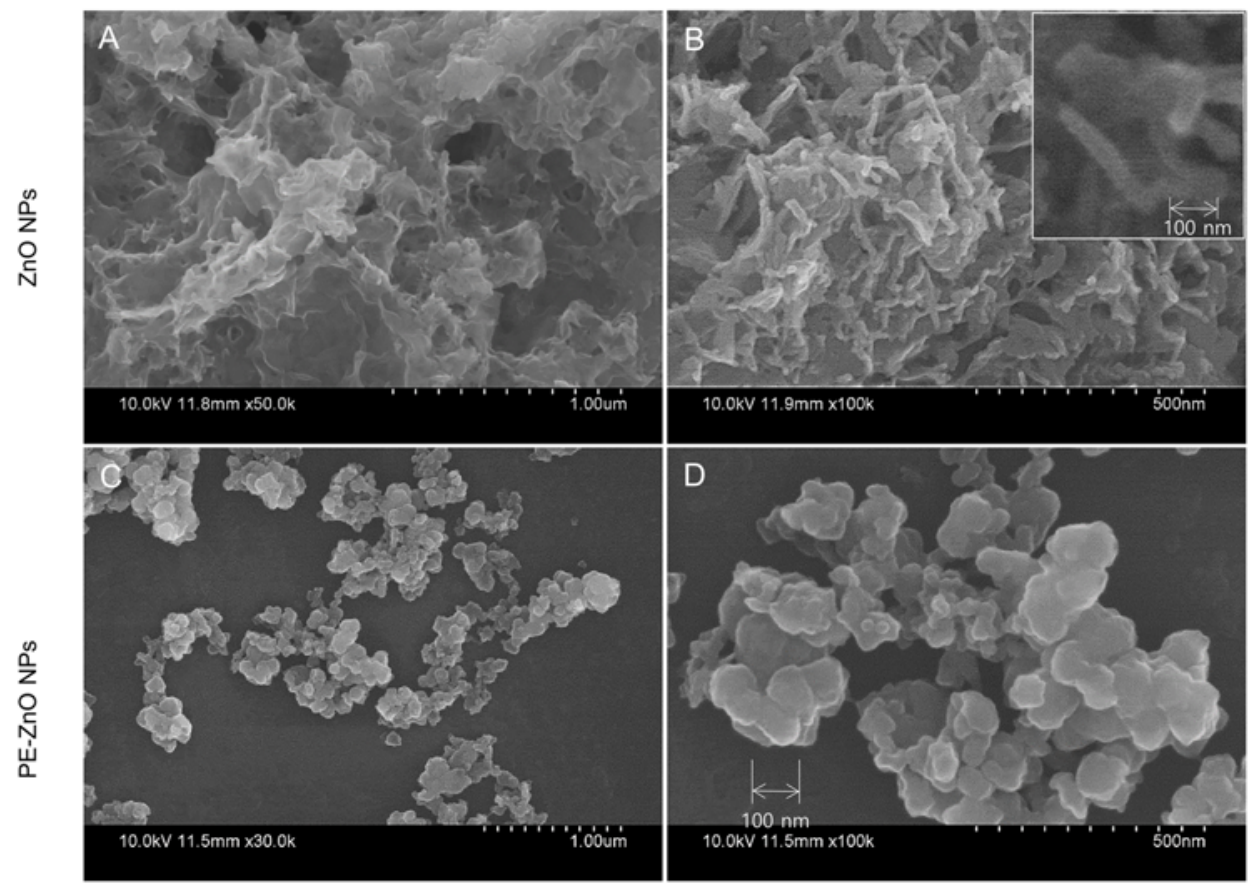

Fig. 2. (A and B) This is a schematic diagram showing the shape of ZnO NPs. (C and D) Pomegranate-zinc oxide nanoparticles (PEZnO NPs) measured by SEM. 

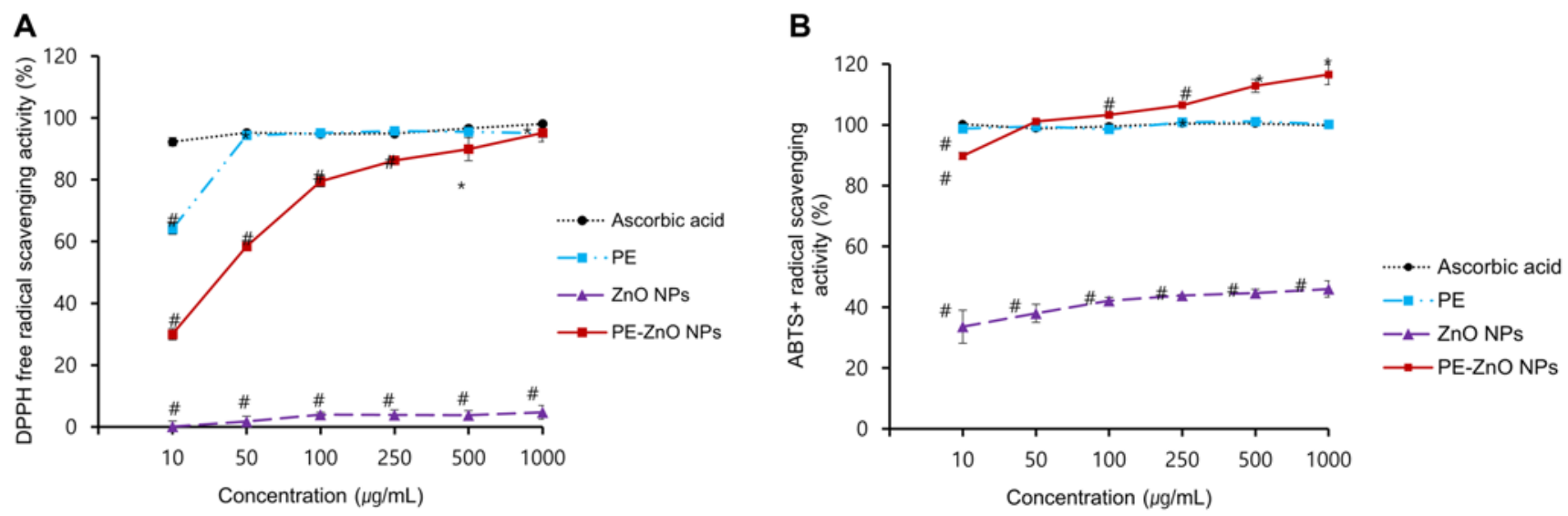

Fig. 3. (A and B) DPPH and $\mathrm{ABTS}^{++}$radical scavenging activity of PE, ZnO NPs, and ZnO-PE NPs. Results are the means $\pm \mathrm{SD},{ }^{*} p<$ $0.05,{ }^{\#} p<0.005$ compared with ascorbic acid (AA).

with bioactive compounds in PE. To note, $\mathrm{ZnO}$ NPs show a broad transmittance peak at $477 \mathrm{~cm}^{-1}$ while characteristic peaks at $585-450 \mathrm{~cm}^{-1}$ noted in the PE-ZnO NPs are not present in PE (Fig. 1B). Based on FT-IR results, functionalization of $\mathrm{PE}$ with $\mathrm{ZnO} \mathrm{NPs}$ was successfully established. In the previous reports, $\mathrm{ZnO}$ NPs were fabricated using extracts of Rubus fairholmianus [18] and Tectona grandis (L.) [41]. Therefore, collected results demonstrate that bioactive compounds in the PE can be used in the functionalization of $\mathrm{ZnO}$ NPs.

Moreover, SEM imaging was applied to characterize the formed nanostructures in PE-ZnO NPs by comparison to $\mathrm{ZnO}$ NPs. Fig. 2A shows the $\mathrm{ZnO}$ NPs as sheets and foamlike nanostructures in the range of $20-100 \mathrm{~nm}$ thickness (Fig. 2A and 2B). However, the PE-ZnO NPs are noted as clusters of nanoplates with varied size in the range of 80$100 \mathrm{~nm}$ (Fig. 2C and 2D). Recently, the biosynthesis of ZnO-NPs using Pongamia pinata, Plectranthus amboinicus, and Vitex negundo also showed agglomerated NPs of different sizes in the range of 80-100 nm [42-44].

\subsection{In vitro antioxidant activity of $P E$ and PE-ZnO NPs}

$\mathrm{PE}$ and PE-ZnO NPs showed an enhanced $\mathrm{ABTS}^{+}$and DPPH radical scavenging activity in a concentrationdependent fashion (Fig. 3A). The percentage scavenging activity of PE and PE-ZnO NPs $(1,000 \mu \mathrm{g} / \mathrm{mL})$ were 95.1 and $95.2 \%$, respectively. $\mathrm{ABTS}^{+}$and $\mathrm{DPPH}$ assays are widely applied as indicators to assess the antioxidant activity of diverse plant extracts via hydrogen donating antioxidants [45]. As a result of measuring $\mathrm{ABTS}^{+}$radical scavenging activity, the PE-ZnO NPs (50 and 1,000 $\mu \mathrm{g} / \mathrm{mL}$ ) exhibited a yield of 101.1 and $116.5 \%$, respectively by comparison to $\mathrm{PE}$ and $\mathrm{ZnO} \mathrm{NPs}$, which showed the better scavenging ability of PE-ZnO NPs against $\mathrm{ABTS}^{+}$(Fig. 3B). Hence, the functionalization of $\mathrm{ZnO}$ NPs with polyphenol compounds present in $\mathrm{PE}$ in $\mathrm{ZnO}(\mathrm{PE}-\mathrm{ZnO})$ was assumed to enhance the $\mathrm{ABTS}^{+}$radical scavenging activity.

\subsection{Cytotoxicity and proliferation rate of PE-ZnO NPs in $\mathrm{HaCaT}$ cells}

A cytotoxicity activity using MTT assay to find the nontoxic concentration of PE-ZnO NPs in HaCaT cells line. Results showed that $\mathrm{ZnO}$ NPs reduced the cell number by $27.2 \%$ at $100 \mu \mathrm{g} / \mathrm{mL}$ concentration by comparison to the control. The result showed that PE and PE-ZnO NPs at $100 \mu \mathrm{g} / \mathrm{mL}$ concentration have 91.3 and $96.3 \%$ of cell viability, respectively. These results indicated that $\mathrm{PE}$ and PE-ZnO NPs are not shown significant toxicity to HaCaT cells (Fig. 4). The ZnO NPs presented photocatalytic toxicity and cytotoxicity in mammalian cells by photo induced effects of noble metal-doped ZnO NPs to produced ROS under UV and visible light [46]. However, the PE-ZnO NPs are not cytotoxic to HaCaT cells, it can be explained

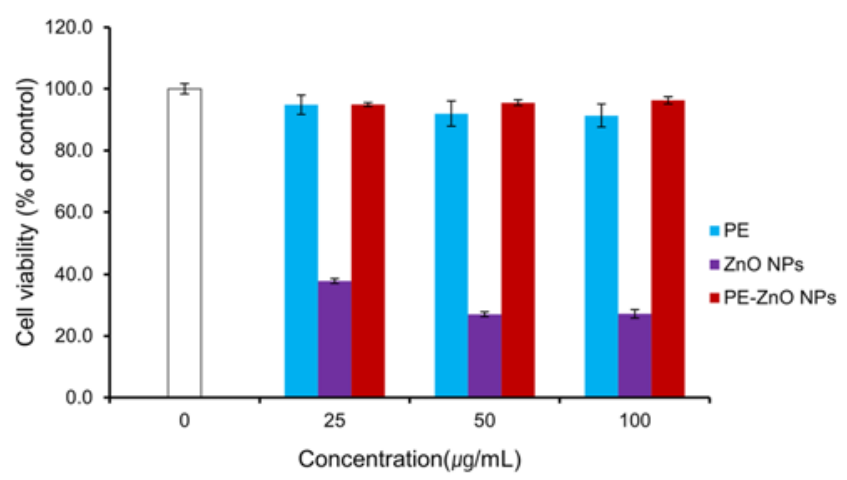

Fig. 4. Effect of PE, ZnO NPs, and PE-ZnO NPs on human skin epithelial keratinocytes $(\mathrm{HaCaT})$ cells viability. The cells were treated with different concentrations of PE-ZnO NPs $(25,50$, and $100 \mu \mathrm{g} / \mathrm{mL}$ ) for $24 \mathrm{~h}$ and then assessed for cell viability by MTT assay. 
that the synthesized PE-ZnO NPs decorated with phytocomponents present in $\mathrm{PE}$, which could reduce the cell toxicity than that of $\mathrm{ZnO}$ NPs (Fig. 4).

\subsection{In vitro antimicrobial activity of PE-ZnO NPs}

Although several species of the Bacillus and Escherichia genus are useful as probiotics, food fermentations, and industrial applications but many of the species from the same genus are also known as pathogens $[47,48]$. Of note, $B$. cereus, gram-positive, facultative anaerobic and sporeforming soil, plant, and food bacteria have been noted for causing. foodborne illnesses, severe nausea, vomiting, and diarrhea [49]. Similarly, B. licheniformis is also a Grampositive and mesophilic bacterium and commonly found in the soil. It is a human pathogen causing skin infections with degradation of $\beta$-keratin [50]. Moreover, E. coli is a gram-negative, facultative anaerobic, rod-shaped, coliform bacterium, which commonly found in the gut of mammals. Although, most of the E. coli strains are non-pathogenic bacteria, but some strains of $E$. coli strains, such as $E$. coli O157:H7, are known to cause serious food poisoning [51]. Therefore, anti-microbial activity of PE-ZnO NPs against such pathogenic bacteria projected the potential and prospective application of nano-materials as non-antibiotic drugs. Therefore, in this study, we tested the antibacterial activity of PE and PE-ZnO NPs at various percentages against $B$. cereus, B. licheniformis, and E. coli by colonyforming unit (CFU) methods.

Herein, the CFU of B. cereus was reduced to $6 \times 10^{5}$ CFU when treated with PE-ZnO NPs $(0.1 \mathrm{and} 1.0 \mathrm{mg} / \mathrm{mL})$ by comparison to $100 \times 10^{5} \mathrm{CFU} / \mathrm{mL}$ of non-treated control, $12 \times 10^{5} \mathrm{CFU} / \mathrm{mL}$ of PE $(0.1$ and $1.0 \mathrm{mg} / \mathrm{mL})$ and $6 \times 10^{5}$ $\mathrm{CFU} / \mathrm{mL}$ of $\mathrm{ZnO}$ NPs $(0.1$ and $1.0 \mathrm{mg} / \mathrm{mL})$ as depicted in Fig. 5. The treatments with PE-ZnO NPs $(1.0 \mathrm{mg} / \mathrm{mL})$ also showed substantial reduction in the growth of $B$. cereus (Fig. 5A and 5B). Moreover, there was no colony formation in B. licheniformis and E. coli treated with 0.1 and $1.0 \mathrm{mg} / \mathrm{mL}$ of PE-ZnO NPs (Fig. 5C-F). Therefore, the synthesized
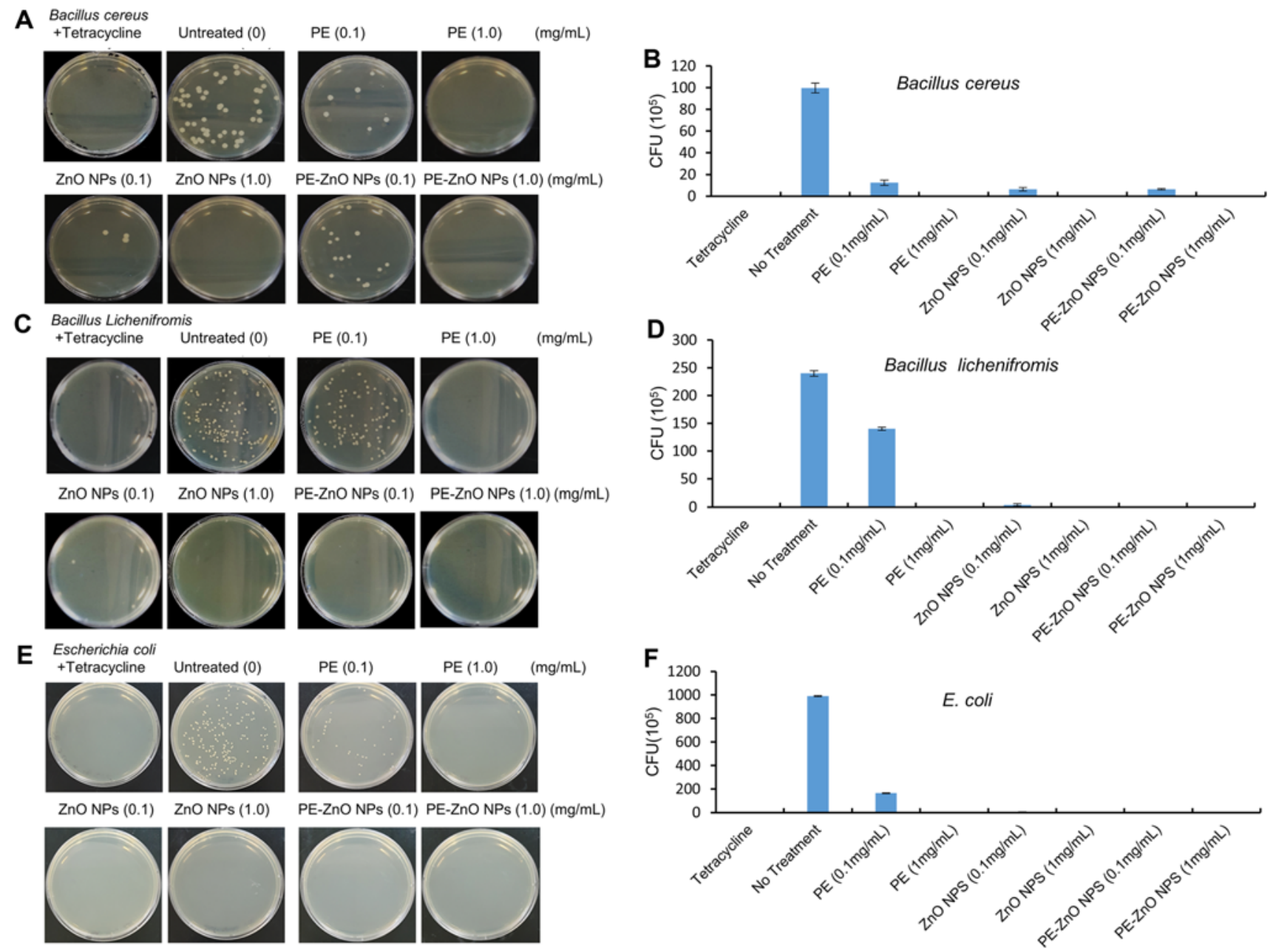

Fig. 5. The antibacterial efficiency of the PE, ZnO NPs, and PE-ZnO NPs against Bacillus cereus, Bacillus licheniformis, and Escherichia coli. The colony-forming units $(\mathrm{CFU} / \mathrm{mL})$ of PE, ZnO NPs, and PE-ZnO NPs. 
PE-ZnO NPs were elucidated with potential antimicrobial activity. Recently, several studies reported that ZnO NPs effectively inhibit bacterial growth [43]. The ZnO NPs streamlines their access via the microbial cell membrane, and hence, to inactivate the protein and bacteria growth [44]. Among the several antimicrobial mechanisms, $\mathrm{ZnO}$ NPs are known to produce ROS and hydrogen peroxides $\left(\mathrm{H}_{2} \mathrm{O}_{2}\right)$. These chemical species can easily inactivate the DNA replication and induce damage in the bacterial cell membrane, resulting in the demolition of bacteria [45]. Bacterial cell degradation proposed of noble metal-doped $\mathrm{ZnO}$ NPs for generating ROS for killing bacterial cells. Hence, the synthesized PE-ZnO NPs were suggested to exhibit superior anti-microbial activity via causing DNA instability and destruction of the bacterial cell membrane. Furthermore, PE-ZnO NPs enhanced the ROS eruption with polyphenolics, leading to antibacterial effects.

Recently, antibiotic resistance microbes (ARM), including multi-drug-resistant (MDR) bacteria, have been increasing globally due to an increased prescription of antibiotic drugs specifically in the COVID-19 pandemic [52]. Therefore, since the PE-ZnO NPs exhibited a wide range of antimicrobial activities, these novel NPs can be used as multifunctional antimicrobial agents function as non-antibiotic drugs. In addition, the PE-ZnO NPs have multifunctional properties with strong ROS scavengers due to the composition of metallic $\mathrm{Zn}$ ions and bioactive compounds.

\section{Conclusion}

In this study, we developed and synthesized the novel organic-ZnO NPs by functionalizing the bioactive compounds in the pomegranate pericarp ethanol extracts (PE) with ZnO NPs. Furthermore, the PE-ZnO NPs exhibited strong radical scavenging activity, suggesting the PE-ZnO NPs would be useful antioxidant agent against ROS-mediated dysfunctions in human cells. Altogether, the synthesized PE-ZnO NPs are organic and metal-based nanoparticles and valuable as multi-target antimicrobial agents in cosmetics and pharmaceuticals.

\section{Acknowledgments}

The authors thank the Core Research Support Center for Natural Products and Medical Materials (CRCNM) at Yeungnam University, Gyeongsan, the Republic of Korea for technical support regarding physiochemical analysis using the Zetasizer Nano ZS (Malvern Panalytical Ltd. Malvern, UK), the FTIR (Fourier Transform Infrared Spectrometer) (PerkinElmer, Inc., Waltham, USA) and SEM
(Scanning Electron Microscope) (SEM S4800, Hitachi, Ltd.).

\section{Funding}

This research was supported by the "LINC+ (Leaders in INdustry-University Co-operation + )" project of the Ministry of Education, Republic of Korea (2021-D-G043-010115).

\section{Disclosure}

The authors declare no conflicts of interest.

No ethical approval or informed consent was required for this study.

\section{References}

1. Sirelkhatim, A., S. Mahmud, A. Seeni, N. H. M. Kaus, L. C. Ann, S. K. M. Bakhori, H. Hasan, and D. Mohamad (2015) Review on zinc oxide nanoparticles: antibacterial activity and toxicity mechanism. Nanomicro Lett. 7: 219-242.

2. Ali, A., A. R. Phull, and M. Zia (2018) Elemental zinc to zinc nanoparticles: Is $\mathrm{ZnO}$ NPs crucial for life? Synthesis, toxicological, and environmental concerns. Nanotechnol. Rev. 7: 413-441.

3. Długosz, O., K. Szostak, A. Staroń, J. Pulit-Prociak, and M. Banach (2020) Methods for reducing the toxicity of metal and metal oxide NPs as biomedicine. Materials. 13: 279.

4. Jiang, J., J. Pi, and J. Cai (2018) The advancing of zinc oxide nanoparticles for biomedical applications. Bioinorg. Chem. Appl. 2018: 1062562.

5. Patra, J. K., G. Das, L. F. Fraceto, E. V. R. Campos, M. del Pilar Rodriguez-Torres, L. S. Acosta-Torres, L. A. Diaz-Torres, R. Grillo, M. K. Swamy, S. Sharma, S. Habtemariam, and H. S. Shin (2018) Nano based drug delivery systems: recent developments and future prospects. J. Nanobiotechnol. 16: 71.

6. Gao, Y., M. A. V. Anand, V. Ramachandran, V. Karthikkumar, V. Shalini, S. Vijayalakshmi, and D. Ernest (2019) Biofabrication of zinc oxide nanoparticles from Aspergillus niger, their antioxidant, antimicrobial and anticancer activity. J. Clust. Sci. 30: 937-946.

7. Román, L. E., J. Huachani, C. Uribe, J. L. Solís, M. M. Gómez, S. Costa, and S. Costa (2019) Blocking erythemally weighted UV radiation using cotton fabrics functionalized with $\mathrm{ZnO}$ nanoparticles in situ. Appl. Surf. Sci. 469: 204-212.

8. Srinivasan, S., S. Srinivasan, V. Ramachandran, R. Murali, V. Vinothkumar, D. Raajasubramanian, and A. Kanagalakshimi (2020) Biogenic metal nanoparticles and their antimicrobial properties. In: S. B. Dhull, P. Chawla, and R. Kaushik (eds.). Nanotechnological Approaches in Food Microbiology. CRC Press, Boca Raton, FL, USA.

9. Siddiqi, K. S., A. Ur Rahman, Tajuddin, and A. Husen (2018) Properties of zinc oxide nanoparticles and their activity against microbes. Nanoscale Res. Lett. 13: 141.

10. Włodarczyk, R. and A. Kwarciak-Kozłowska (2021) Nanoparticles from the cosmetics and medical industries in legal and environmental aspects. Sustainability. 13: 5805.

11. Jeevanandam, J., A. Barhoum, Y. S. Chan, A. Dufresne, and M. K. Danquah (2018) Review on nanoparticles and nanostructured materials: history, sources, toxicity and regulations. Beilstein J. Nanotechnol. 9: 1050-1074. 
12. Katz, L. M., K. Dewan, and R. L. Bronaugh (2015) Nanotechnology in cosmetics. Food Chem. Toxicol. 85: 127-137.

13. Smijs, T. G. and S. Pavel (2011) Titanium dioxide and zinc oxide nanoparticles in sunscreens: focus on their safety and effectiveness. Nanotechnol. Sci. Appl. 4: 95-112.

14. Twilley, D., D. Moodley, H. Rolfes, I. Moodley, L. J. McGaw, B. Madikizela, B. Summers, L. Raaff, M. Lategan, L. Kgatuke, E. C. Mabena, N. Lall (2021) Ethanolic extracts of South African plants, Buddleja saligna Willd. and Helichrysum odoratissimum (L.) Sweet, as multifunctional ingredients in sunscreen formulations. S. Afr. J. Bot. 137: 171-182.

15. Thi, T. U. D., T. T. Nguyen, Y. D. Thi, K. H. T. Thi, B. T. Phan, and K. N. Pham (2020) Green synthesis of $\mathrm{ZnO}$ nanoparticles using orange fruit peel extract for antibacterial activities. RSC Adv. 10: 23899-23907.

16. Luque, P. A., C. A. Soto-Robles, O. Nava, C. M. Gomez-Gutierrez, A. Castro-Beltran, H. E. Garrafa-Galvez, A. R. Vilchis-Nestor, and A. Olivas (2018) Green synthesis of zinc oxide nanoparticles using Citrus sinensis extract. J. Mater. Sci. Mater. Electron. 29: 9764-9770.

17. Nava, O. J., C. A. Soto-Robles, C. M. Gómez-Gutiérrez, A. R. Vilchis-Nestor, A. Castro-Beltrán, A. Olivas, and P. A. Luque (2017) Fruit peel extract mediated green synthesis of zinc oxide nanoparticles. J. Mol. Struct. 1147: 1-6.

18. Rajendran, N. K., B. P. George, N. N. Houreld, and H. Abrahamse (2021) Synthesis of zinc oxide nanoparticles using Rubus fairholmianus root extract and their activity against pathogenic bacteria. Molecules. 26: 3029.

19. Qin, G., C. Xu, R. Ming, H. Tang, R. Guyot, E. M. Kramer, Y. Hu, X. Yi, Y. Qi, X. Xu, Z. Gao, H. Pan, J. Jian, Y. Tian, Z. Yue, and $\mathrm{Y}$. Xu (2017) The pomegranate (Punica granatum L.) genome and the genomics of punicalagin biosynthesis. Plant $J$. 91: 1108-1128.

20. Fahmy, H., N. Hegazi, S. El-Shamy, and M. A. Farag (2020) Pomegranate juice as a functional food: a comprehensive review of its polyphenols, therapeutic merits, and recent patents. Food Funct. 11: 5768-5781.

21. Viuda-Martos, M., J. Fernández-López, and J. A. Pérez-Álvarez (2010) Pomegranate and its many functional components as related to human health: a review. Compr. Rev. Food Sci. Food Saf. 9: 635-654.

22. Fischer, U. A., R. Carle, and D. R. Kammerer (2011) Identification and quantification of phenolic compounds from pomegranate (Punica granatum L.) peel, mesocarp, aril and differently produced juices by HPLC-DAD-ESI/MSn. Food Chem. 127: 807-821.

23. Caruso, A., A. Barbarossa, A. Tassone, J. Ceramella, A. Carocci, A. Catalano, G. Basile, A. Fazio, D. Iacopetta, C. Franchini, and M. S. Sinicropi (2020) Pomegranate: nutraceutical with promising benefits on human health. Appl. Sci. 10: 6915.

24. Khajebishak, Y., L. Payahoo, M. Alivand, and B. Alipour (2019) Punicic acid: A potential compound of pomegranate seed oil in Type 2 diabetes mellitus management. J. Cell. Physiol. 234: 2112-2120.

25. Xu, K. Z. Y., C. Zhu, M. S. Kim, J. Yamahara, and Y. Li (2009) Pomegranate flower ameliorates fatty liver in an animal model of type 2 diabetes and obesity. J. Ethnopharmacol. 123: 280-287.

26. Stowe, C. B. (2011) The effects of pomegranate juice consumption on blood pressure and cardiovascular health. Complement. Ther. Clin. Pract. 17: 113-115.

27. Aviram, M., L. Dornfeld, M. Kaplan, R. Coleman, D. Gaitini, S. Nitecki, A. Hofman, M. Rosenblat, N. Volkova, D. Presser, J. Attias, T. Hayek, and B. Fuhrman (2002) Pomegranate juice flavonoids inhibit low-density lipoprotein oxidation and cardiovascular diseases: studies in atherosclerotic mice and in humans. Drugs Exp. Clin. Res. 28: 49-62.
28. Lansky, E. P. and R. A. Newman (2007) Punica granatum (pomegranate) and its potential for prevention and treatment of inflammation and cancer. J. Ethnopharmacol. 109: 177-206.

29. Larrosa, M., A. González-Sarrías, M. J. Yáñez-Gascón, M. V. Selma, M. Azorín-Ortuño, S. Toti, F. Tomás-Barberán, P. Dolara, and J. C. Espín (2010) Anti-inflammatory properties of a pomegranate extract and its metabolite urolithin-A in a colitis rat model and the effect of colon inflammation on phenolic metabolism. J. Nutr. Biochem. 21: 717-725.

30. Wang, L. and M. Martins-Green (2014) Pomegranate and its components as alternative treatment for prostate cancer. Int. J. Mol. Sci. 15: 14949-14966.

31. Hajleh, M. A. and E. A. S. Al-Dujaili (2016) Anti-cancer activity of pomegranate and its biophenols; general review. EC Nutr. 6: 28-52.

32. Howell, A. B. and D. H. D'Souza (2013) The pomegranate: effects on bacteria and viruses that influence human health. Evid. Based Complement. Alternat. Med. 2013: 606212.

33. Khaleghnezhad, V., A. R. Yousefi, A. Tavakoli, and B. Farajman (2019) Interactive effects of abscisic acid and temperature on rosmarinic acid, total phenolic compounds, anthocyanin, carotenoid and flavonoid content of dragonhead (Dracocephalum moldavica L.). Sci. Hortic. 250: 302-309.

34. Re, R., N. Pellegrini, A. Proteggente, A. Pannala, M. Yang, and C. Rice-Evans (1999) Antioxidant activity applying an improved ABTS radical cation decolorization assay. Free Radic. Biol. Med. 26: 1231-1237.

35. Blois, M. S. (1958) Antioxidant determinations by the use of a stable free radical. Nature. 181: 1199-1200.

36. Kandylis, P. and E. Kokkinomagoulos (2020) Food applications and potential health benefits of pomegranate and its derivatives. Foods. 9: 122.

37. Akhtar, S., T. Ismail, D. Fraternale, and P. Sestili (2015) Pomegranate peel and peel extracts: Chemistry and food features. Food Chem. 174: 417-425.

38. Derakhshan, Z., M. Ferrante, M. Tadi, F. Ansari, A. Heydari, M. S. Hosseini, G. O. Conti, and E. K. Sadrabad (2018) Antioxidant activity and total phenolic content of ethanolic extract of pomegranate peels, juice and seeds. Food Chem.Ttoxicol. 114: 108-111.

39. Ardekani, M. R. S., M. Hajimahmoodi, M. R. Oveisi, N. Sadeghi, B. Jannat, A. M. Ranjbar, N. Gholam, and T. Moridi (2011) Comparative antioxidant activity and total flavonoid content of Persian pomegranate (Punica granatum L.) cultivars. Iran. J. Pharm. Res. 10: 519-524.

40. Del Buono, D., A. Di Michele, F. Costantino, M. Trevisan, and L. Lucini (2021) Biogenic $\mathrm{ZnO}$ nanoparticles synthesized using a novel plant extract: Application to enhance physiological and biochemical traits in maize. Nanomaterials. 11: 1270.

41. Senthilkumar, N., E. Nandhakumar, P. Priya, D. Soni, M. Vimalane, and I. V. Potheher (2017) Synthesis of $\mathrm{ZnO}$ nanoparticles using leaf extract of Tectona grandis (L.) and their anti-bacterial, anti-arthritic, anti-oxidant and in vitro cytotoxicity activities. New J. Chem. 41: 10347-10356.

42. Sundrarajan, M., S. Ambika, and K. Bharathi (2015) Plantextract mediated synthesis of $\mathrm{ZnO}$ nanoparticles using Pongamia pinnata and their activity against pathogenic bacteria. $A d v$. Powder Technol. 26: 1294-1299.

43. Fu, L. and Z. Fu (2015) Plectranthus amboinicus leaf extractassisted biosynthesis of $\mathrm{ZnO}$ nanoparticles and their photocatalytic activity. Ceram. Int. 41: 2492-2496.

44. Ambika, S. and M. Sundrarajan (2015) Green biosynthesis of $\mathrm{ZnO}$ nanoparticles using Vitex negundo L. extract: spectroscopic investigation of interaction between $\mathrm{ZnO}$ nanoparticles and human serum albumin. J. Photochem. Photobiol. B. 149: 143-148. 45. Sowndhararajan, K. and S. C. Kang (2013) Free radical 
scavenging activity from different extracts of leaves of Bauhinia vahlii Wight \& Arn. Saudi J. Biol. Sci. 20: 319-325.

46. Liao, C., Y. Jin, Y. Li, and S. C. Tjong (2020) Interactions of zinc oxide nanostructures with mammalian cells: Cytotoxicity and photocatalytic toxicity. Int. J. Mol. Sci. 21: 6305.

47. Chmielewska, A. and H. Szajewska (2010) Systematic review of randomised controlled trials: probiotics for functional constipation. World J. Gastroenterol. 16: 69-75.

48. Song, D., S. Ibrahim, and S. Hayek (2012) Recent application of probiotics in food and agricultural science. In: E. Rigobelo (ed.). Probiotics. IntechOpen.

49. Stenfors Arnesen, L. P., A. Fagerlund, and P. E. Granum (2008)
From soil to gut: Bacillus cereus and its food poisoning toxins. FEMS Microbiol. Rev. 32: 579-606.

50. Haydushka, I. A., N. Markova, V. Kirina, and M. Atanassova (2012) Recurrent sepsis due to Bacillus licheniformis. J. Glob. Infect. Dis. 4: 82-83.

51. Croxen, M. A., R. J. Law, R. Scholz, K. M. Keeney, M. Wlodarska, and B. B. Finlay (2013) Recent advances in understanding enteric pathogenic Escherichia coli. Clin. Microbiol. Rev. 26: 822-880.

52. Pelfrene, E., R. Botgros, and M. Cavaleri (2021) Antimicrobial multidrug resistance in the era of COVID-19: a forgotten plight? Antimicrob. Resist. Infect. Control. 10: 21.

Publisher's Note Springer Nature remains neutral with regard to jurisdictional claims in published maps and institutional affiliations. 